\title{
Formal Validation of Pattern Matching Code
}

\author{
Claude Kirchner, Pierre-Etienne Moreau, Antoine Reilles \\ INRIA \& LORIA, INRIA \& LORIA CNRS \& LORIA \\ Nancy, France \\ First.Last@loria.fr
}

July 27, 2006

\begin{abstract}
When addressing the formal validation of generated software, two main alternatives consist either to prove the correctness of compilers or to directly validate the generated code. Here, we focus on directly proving the correctness of compiled code issued from powerful pattern matching constructions typical of ML like languages or rewrite based languages such as ELAN, Maude or Tom. In this context, our first contribution is to define a general framework for anchoring algebraic pattern-matching capabilities in existing languages like C, Java or ML. Then, using a just enough powerful intermediate language, we formalize the behavior of compiled code and define the correctness of compiled code with respect to pattern-matching behavior. This allows us to prove the equivalence of compiled code correctness with a generic first-order proposition whose proof could be achieved via a proof assistant or an automated theorem prover. We then extend these results to the multi-match situation characteristic of the ML like languages. The whole approach has been implemented on top of the Tom compiler and used to validate the syntactic matching code of the Tom compiler itself.
\end{abstract}

\section{Introduction}

Even if we know, since the beginning of the computer science era, that proving program correctness is profoundly difficult, the quest of software security and dependability due to the general digitalization of most human activities and process control makes this goal both inescapable and extremely important to reach.

When we deal with the previous problem, we should address the whole software conception process that we can reduce, quite schematically, to the following steps: (i) get an informal specification of the software functionalities, (ii) get a formal description of the algorithms assumed to fulfill the informal specification, (iii) get a high-level program implementation of these algorithms, (iv) get a low level program implementation of these programs, (v) get a model of the running hardware.

In this work, we restrict our interest to step (iv) and to high-level languages pattern-matching features. Therefore we address the specific problem of proving the correctness of compiled code issued from patternmatching constructions appearing in high-level programming languages.

Verifiable - compiler versus compiled - code The question of compiler correctness, that is to preserve the properties of the input like its semantics and meta-properties of the underlying algorithm as its termination or the respect of heap invariants, is as old as the first compiler implementations. This is a very challenging goal since it consists in proving that any valid input will be correctly compiled. Furthermore, this proof has to be done every time the implementation of the compiler is modified and moreover, the compiler has itself to be compiled.

Much efforts have been done on proving correctness of parts and sometimes even complete compilers either manually [?, ?, ?, ?] or with the help of a proof assistant. But, it is still today mostly out of reach 
to prove that a program has been correctly compiled. In practice, programmers (and therefore applications) totally rely on the compilers: until one runs the program, we have no idea if the compiler has compiled the program correctly. Even extensively testing the program of course offers no guarantees. So, currently the programmer very often must blindly trust the compiler. But, most of largely used $\mathrm{C}$ and Fortran compilers very infrequently generate incorrect code: they are some of the most reliable software tools available. This is due to the large number of developers working to make these compilers correct, as well as the very large number of users who use these compiler, and thus contribute to their debugging. But, when designing a compiler for a new high-level language, the situation is less comfortable: on one side the number of users and written applications is small, on the other side, the introduction of new high level constructs put a lot on the compiler, and so make it even more complex to write. Since the consequences of an incorrect compiler are disastrous (all compiled programs are potentially faulty), this situation contributes to make users less confident in new languages and compiler implementations.

In this paper, we are concerned by a quite different approach consisting in proving automatically the correctness of the compiled code. This "skeptical" approach of the code issued from a compiler allows to deal with two kind of mis-behavior: one is due to the classical presence of an unintentional bug of the compiler. The second one concern intended hidden-behavior that could be introduced with malicious intention.

Therefore, assuming a high-level program given as input, we are considering the compiler as a black box escaping our control and we are searching to prove that the generated code is, on its own, correct. This is typical of the seminal work of [?] and more recently of the so called translation validation [?, ?]. A comparable approach presented in [?] is called credible compilation and able to handle pointers in the source program. Note that this is different from the so called proof-carrying code method [?, ?] which is not intended to prove the compiled program to be correct with respect to the source code, but rather on proving certain properties on the output program, such as type safety, memory safety, or the respect of a certain safety invariant.

Matching power Rewriting and pattern-matching are of general use in mathematics and informatics to describe computation as well as deduction. They are central in systems making the notion of rule an explicit and first class object, like expert and business systems (JRule), programming languages based on equational logic (OBJ) or the rewriting calculus (ELAN) or logic (Maude), functional, possibly logic, programming (ML, Haskell, Curry, Teyjus) and model checkers (Murphi). They are also recognized as crucial components of proof assistants (Coq, Isabelle) and theorem provers for expressing computation as well as strategies.

Since pattern-matching is directly related to the structure of objects and therefore is a very natural programming language feature, it is a first class citizen of functional languages like ML or Haskell and has been considered recently as a useful add-on facility in object programming languages [?]. This is formally backed up by works like [?] and particularly well-suited when describing various transformations of structured entities like, for example, trees/terms, hierarchized objects, and XML documents.

In this context, we are developing the Tom system [?] which provides a generic way to integrate matching power in existing programming languages like Java, C or ML. For example, when using Java as the host language, the sum of two integers can be described in Tom as follows:

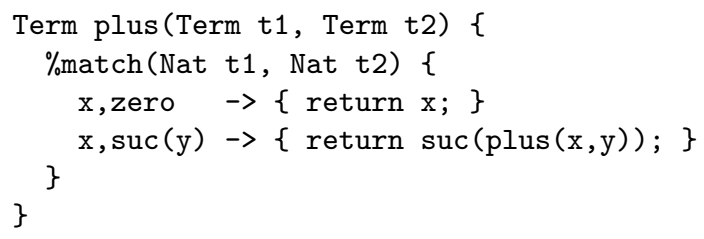


In this example, given two terms $t_{1}$ and $t_{2}$ that represent Peano integers, the evaluation of plus computes their sum. This is implemented by pattern-matching: $t_{1}$ is matched by the variable $x, t_{2}$ is possibly matched by one of the two patterns zero or suc(y). When zero matches $t_{2}$, the result of the addition is $x$ (where $x$ has been instantiated into $t_{1}$ via matching). When $s u c(y)$ matches $t_{2}$, this means that $t_{2}$ is rooted by a suc symbol: the subterm $y$ is added to $x$ and the successor of this number is returned. This definition of plus is given in a functional style, but now the plus function can be used elsewhere in a Java program to perform addition.

The general architecture of Tom, depicted as follows,

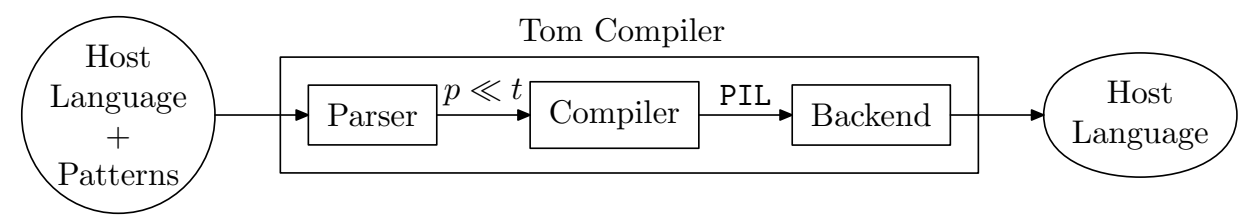

enlightens that a generic matching problem $p \ll t$ is compiled into an intermediate language code (PIL) which we would like to compute a substitution $\sigma$ iff $\sigma(p)=t$. As explained in [?], implementing a language (possibly domain-specific) as an extension of an existing host language has several advantages. First, we benefit of the existing functionalities and we do not have to re-implement common language constructs. Second, the extensions themselves only need to be transformed to the point where they are expressible in the host language. Third, existing infrastructure can be reused. All these factors result into lower implementation costs and decrease the risk of building an incorrect compiler.

So, in this work we focus on proving the correctness of compiled code issued from pattern-matching constructions, and to the best of our knowledge, this is the first attempt to do so. Other works about pattern-matching compilation address in particular data abstraction e.g. [?], or optimizations for run-time efficiency or code size e.g. [?, ?].

Roadmap of the paper When considering the notion of pattern-matching, we consider a term datastructure against which some patterns are matched. Since the host language is not fixed and could be typically either C, Java or ML, the data-model is unknown. We therefore introduce in Section 2, the notion of formal anchor which formally describes the relationship between the host language data-model and the algebraic notion of term and pattern.

In our language, the host language is also generic, so we have to consider an abstraction which describes the minimal set of functionality the host language should have to express the compilation of pattern-matching. This abstraction is called the intermediate language (PIL) and we define its syntax and its big-step semantics in Section 3 ,

Then Section 4 uses the proposed framework to define the correctness of a single pattern compilation and to show how this correctness can be reduced to the validation of a first-order proposition.

This result is then extended in Section 5 to support Caml or Tom multi-match constructs, and before concluding, Section 7 provides details about the implementation of these concepts in the current version of Tom.

\section{Formal anchor}

When considering the problem of proving that the behavior of a program is compatible with its semantics, we have to consider two kinds of entities. On the one side, we consider algebraic constructions, such as ground terms $(t \in \mathcal{T}(\mathcal{F}))$, patterns $(p \in \mathcal{T}(\mathcal{F}, \mathcal{X}))$, and matching problems $(p \ll t$, with $p \in \mathcal{T}(\mathcal{F}, \mathcal{X})$ and $t \in \mathcal{T}(\mathcal{F})$ ). On the other side, we consider programs, expressed in the PIL intermediate language, which are supposed to solve matching problems. We also consider data which are supposed to represent a term or a pattern. In this section, we define the notions of representation and formal anchor which define the link between algebraic entities and considered data. 


\subsection{Preliminary concepts}

We assume the reader to be familiar with the basic definitions of first order term given, in particular, in [?]. We briefly recall or introduce notation for a few concepts that will be used along this paper.

A signature $\mathcal{F}$ is a set of function symbols, each one associated to a natural number by the arity function $(\operatorname{ar}: \mathcal{F} \rightarrow \mathbb{N}) . \mathcal{F}_{n}$ is the subset of function symbols having $n$ for arity, $\mathcal{F}_{n}=\{f \in \mathcal{F} \mid \operatorname{ar}(f)=n\}$.

$\mathcal{T}(\mathcal{F}, \mathcal{X})$ is the set of terms built from a given finite set $\mathcal{F}$ of function symbols and a denumerable set $\mathcal{X}$ of variables. A term $t$ is said to be linear if no variable occurs more than once in $t$. Positions in a term are represented as sequences of integers and denoted by Greek letters $\epsilon, \nu$. The empty sequence $\epsilon$ denotes the position associated to the root, and it is called the root (or top) position. The subterm of $t$ at position $\nu$ is denoted $t_{\mid \nu}$. Symb $(t)$ is a partial function from $\mathcal{T}(\mathcal{F}, \mathcal{X})$ to $\mathcal{F}$, which associates to each term $t$ its root symbol $f \in \mathcal{F}$.

The set of variables occurring in a term $t$ is denoted by $\mathcal{V} a r(t)$. If $\mathcal{V}$ ar $(t)$ is empty, $t$ is called a ground term and $\mathcal{T}(\mathcal{F})$ is the set of ground terms.

Two ground terms $t$ and $u$ of $\mathcal{T}(\mathcal{F})$ are equal, and we note $t=u$, when, for some function symbol $f$, $\mathcal{S} y m b(t)=\operatorname{Symb}(u)=f, f \in \mathcal{F}_{n}, t=f\left(t_{1}, \ldots, t_{n}\right), u=f\left(u_{1}, \ldots, u_{n}\right)$, and $\forall i \in[1 . . n], t_{i}=u_{i}$.

A substitution $\sigma$ is an assignment from $\mathcal{X}$ to $\mathcal{T}(\mathcal{F})$, written, when its domain is finite, $\sigma=\left\{x_{1} \mapsto\right.$ $\left.t_{1}, \ldots, x_{k} \mapsto t_{k}\right\}$. It uniquely extends to an endomorphism $\sigma^{\prime}$ of $\mathcal{T}(\mathcal{F}, \mathcal{X}): \sigma^{\prime}(x)=\sigma(x)$ for each variable $x \in \mathcal{X}, \sigma^{\prime}\left(f\left(t_{1}, \ldots, t_{n}\right)\right)=f\left(\sigma^{\prime}\left(t_{1}\right), \ldots, \sigma^{\prime}\left(t_{n}\right)\right)$ for each function symbol $f \in \mathcal{F}_{n}$.

Given a pattern $p \in \mathcal{T}(\mathcal{F}, \mathcal{X})$ and a ground term $t \in \mathcal{T}(\mathcal{F}), p$ matches $t$, written $p \ll t$, if and only if there exists a substitution $\sigma$ such that $\sigma(p)=t$. Its negation is written $p \nless t$.

\subsection{Object representation}

Definition 1 Given a tuple composed of a signature $\mathcal{F}$, a set of variables $\mathcal{X}$, booleans $\mathbb{B}$ and integers $\mathbb{N}$, given sets $\Omega_{\mathcal{F}}, \Omega_{\mathcal{X}}, \Omega_{\mathcal{T}}, \Omega_{\mathbb{B}}$, and $\Omega_{\mathbb{N}}$, we consider a family of representation functions \ulcorner\urcorner that map:

- function symbols $f \in \mathcal{F}$ to elements of $\Omega_{\mathcal{F}}$, denoted $\ulcorner f\urcorner$,

- variables $v \in \mathcal{X}$ to elements of $\Omega_{\mathcal{X}}$, denoted $\ulcorner v\urcorner$,

- ground terms $t \in \mathcal{T}(\mathcal{F})$ to elements of $\Omega_{\mathcal{T}}$, denoted $\ulcorner t\urcorner$,

- booleans $b \in \mathbb{B}=\{\top, \perp\}$ to elements of $\Omega_{\mathbb{B}}$, denoted $\ulcorner b\urcorner$,

- natural numbers $n \in \mathbb{N}$ to elements of $\Omega_{\mathbb{N}}$, denoted $\ulcorner n\urcorner$.

In other words, the representation function \ulcorner\urcorner maps algebraic entities (from $\mathcal{F}, \mathcal{X}, \mathcal{T}(\mathcal{F}), \mathbb{B}$, and $\mathbb{N}$ ) to objects manipulable by the intermediate language PIL (elements of $\Omega_{\mathcal{F}}, \Omega_{\mathcal{X}}, \Omega_{\mathcal{T}}, \Omega_{\mathbb{B}}$, and $\Omega_{\mathbb{N}}$ ). We note $\ulcorner\mathcal{T}(\mathcal{F})\urcorner$ the set containing the representations of terms: $\ulcorner\mathcal{T}(\mathcal{F})\urcorner=\{\ulcorner t\urcorner \mid t \in \mathcal{T}(\mathcal{F})\}$, and we therefore have $\ulcorner\mathcal{T}(\mathcal{F})\urcorner \subseteq \Omega_{\mathcal{T}}$.

Example 1 Let us consider $\mathcal{F}=\{e, s\}$ (with ar $(e)=0$ and $\operatorname{ar}(s)=1$ ), and the function \ulcorner\urcorner such that $\ulcorner e\urcorner=0 \in \Omega_{\mathcal{F}},\ulcorner s\urcorner=1 \in \Omega_{\mathcal{F}}$. \ulcorner\urcorner maps the symbols $e$ and $s$ respectively to "machine integers" 0 and 1 (i.e. the notion of integer in the intermediate language), where we assume an infinite memory. Similarly, $\ulcorner$ can be extended to map the constant $e \in \mathcal{T}(\mathcal{F})$ to $0\left(\ulcorner e\urcorner=0 \in \Omega_{\mathcal{T}}\right)$, and any term of the form $s(x)$ to the result of the addition of 1 and the representation of $x\left(\ulcorner s(x)\urcorner=1+\ulcorner x\urcorner \in \Omega_{\mathcal{T}}\right)$.

This representation is a way to map Peano integers to "machine integers". Another well-known representation is the encoding of algebraic terms into e.g. n-ary trees. 


\subsection{Object mapping}

In Definition 1, the notion of representation mapping has been introduced to establish a correspondence between algebraic objects and their representation in the intermediate language. However, we did not put any constraint on the representation of objects. In particular, the function \ulcorner\urcorner does not necessarily preserve structural properties of algebraic objects (all terms could for example be represented by a unique constant).

Definition 2 Given a tuple $\langle\mathcal{F}, \mathcal{X}, \mathcal{T}(\mathcal{F}), \mathbb{B}, \mathbb{N}\rangle$, a representation function \ulcorner\urcorner , and the mappings eq : $\Omega_{\mathcal{T}} \times$ $\Omega_{\mathcal{T}} \rightarrow \Omega_{\mathbb{B}}$, is_fsym $: \Omega_{\mathcal{T}} \times \Omega_{\mathcal{F}} \rightarrow \Omega_{\mathbb{B}}$, and subterm $_{f}: \Omega_{\mathcal{T}} \times \Omega_{\mathbb{N}} \rightarrow \Omega_{\mathcal{T}}(f \in \mathcal{F})$. A formal anchor is a mapping \lceil\rceil$: \mathcal{T}(\mathcal{F}) \rightarrow\ulcorner\mathcal{T}(\mathcal{F})\urcorner$ such that the structural properties of $\mathcal{T}(\mathcal{F})$ are preserved, in $\ulcorner\mathcal{T}(\mathcal{F})\urcorner$, by the semantics of eq, is_fsym, and subterm $_{f}$.

$\forall t, t_{1}, t_{2} \in \mathcal{T}(\mathcal{F}), \forall f \in \mathcal{F}, \forall i \in[1$. ar $(f)]$ we have:

$$
\begin{aligned}
& \mathrm{eq}\left(\left\lceil t_{1}\right\rceil,\left\lceil t_{2}\right\rceil\right) \equiv\left\lceil t_{1}=t_{2}\right\rceil \\
& \text { is_f } \operatorname{sym}(\lceil t\rceil,\lceil f\rceil) \equiv\lceil\operatorname{S} y m b(t)=f\rceil \\
& \text { subterm }_{f}(\lceil t\rceil,\lceil i\rceil) \equiv\left\lceil t_{\mid i}\right\rceil \text { if } \mathcal{S} y m b(t)=f
\end{aligned}
$$

In the following, we always consider that the representation function is also a formal anchor. Therefore, from now on, the notation \ulcorner\urcorner denotes representations that are also formal anchors.

Example 2 In $C$ or Java like, the notion of term can be implemented by a record (sym: integer, sub:array of term), where the first slot (sym) denotes the top symbol, and the second slot (sub) corresponds to the subterms. It is easy to check that the following definitions of eq, is_f $\mathbf{s y m}$, and subterm $_{f}$ (where $=$ denotes an atomic equality) provide a formal anchor for $\mathcal{T}(\mathcal{F})$ :

$$
\begin{aligned}
\text { eq }\left(t_{1}, t_{2}\right) \triangleq & t_{1} . \text { sym }=t_{2} . \text { sym } \wedge \forall i \in\left[1 . . \operatorname{ar}\left(t_{1} . \text { sym }\right)\right], \\
& \text { eq }\left(t_{1} \cdot \operatorname{sub}[i], t_{2} . \operatorname{sub}[i]\right) \\
\text { is_f } \operatorname{sym}(t, f) \triangleq & t . \operatorname{sym}=f \\
\operatorname{subterm}_{f}(t, i) \triangleq & t . \operatorname{sub}[i] \text { if } t . \operatorname{sym}=f \text { and } i \in[1 . . \operatorname{ar}(f)]
\end{aligned}
$$

Defining a correct formal anchor is a key point to allow for the formal verification of the pattern matching code. But since this can be quite technical, we use in practice an external tool which generates for us the mapping for a given signature, as described in Section 7 .

\section{Intermediate language}

We now describe the syntax of PIL, introduce the notion of environment, and give a formal big-step semantics $\left(\mapsto_{b s}\right)$ to PIL. Informally, this intermediate language is a subset of $C \cap$ Java $\cap$ ML that is expressive enough to describe pattern matching procedures. This language is very close to the host language fragment it will be translated into at the end of the compilation process, and involves only a renaming of the syntactic constructions, so that proving this part of the compilation process should not present difficulties.

\subsection{Syntax}

Given $\mathcal{F}, \mathcal{X}, \mathcal{T}(\mathcal{F}), \mathbb{B}, \mathbb{N}$, eq, is_fsym, subterm, and a formal anchor \ulcorner\urcorner as defined above, the syntax of the intermediate language PIL is defined in Figure 1 .

The set of terms $\langle$ term $\rangle$ is built over the representation of $\mathcal{T}(\mathcal{F})$, and the construct subterm which retrieves the $i^{t h}$ child of a given term. The set of expressions $\langle$ bexpr $\rangle$ contains the representation of booleans, as well as two predicates: eq which compares two terms, and is_fsym which checks that a given term is rooted by a particular symbol given in argument. The set of instructions $\langle i n s t r\rangle$ contains only 4 instructions: 


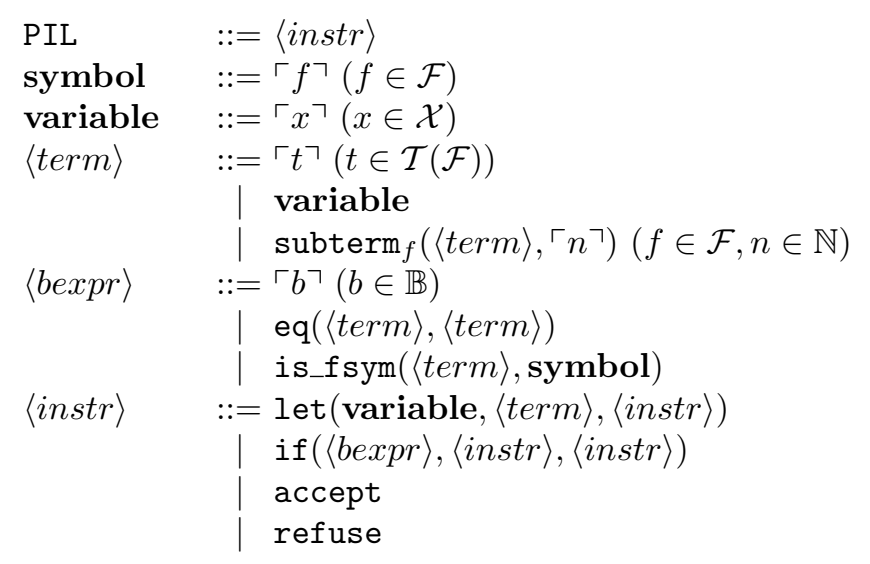

Figure 1: Syntax of the intermediate language PIL

let and if correspond respectively to the assignment and the if-then-else test. We consider here that it is forbidden to assign a same variable twice. We use an if then else construct instead of the switch construct usually used to compile pattern matching because we want the generated matching algorithm to be independent of the mapping and the way terms are effectively represented. The is_fsym expression allows to "query" a term without the need to have function symbols as objects directly manipulated by the host language, providing more abstraction. accept and refuse are two special instructions aimed to approximate the body part of a function defined by pattern matching. In this work, since we focus on pattern matching, we only need two instructions to put the execution in a given state (accept or refuse), which denotes whether the pattern matches the subject or not.

Such a program may contain some free variables (variables which are not bound in the program by a let construct). They represent the input of the program, in our case the terms the pattern matching algorithm will try to match against. We call such variable input variable.

Assumption A. In the following, we consider that a program is evaluated in an environment where all its free variables are instantiated by a value, i.e. a term representation.

Example 3 Given a signature $\mathcal{F}=\{a, f\}$ and a set of variables $\mathcal{X}=\{s, x\}$, a possible compilation of $f(x) \ll s$ is:

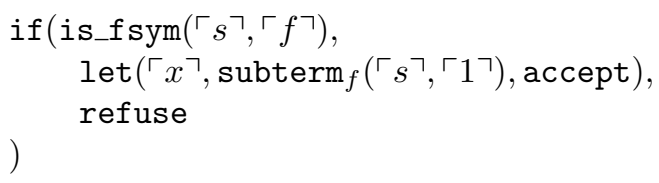

This program is evaluated in an environment which assigns a term representation to the free variable $\ulcorner$ s . This program checks that the root symbol of s corresponds to the representation of $f$. When it is the case, the first subterm of $s$ is assigned to a variable $x$, and the program goes into the accept state. Otherwise, it goes into the refuse state.

On this example, it is easy to convince ourselves that the program goes into the accept state if and only if the pattern effectively matches the subject. Our goal here consists to get a formal proof of this property.

Notation. For sake of correctness, mathematical objects $(\mathbb{B}, \mathbb{N}$, and $\mathcal{X})$ have to be distinguished from their representation. However, since most of programming languages support the notion of boolean, integer and variable, when there is no ambiguity, we note $\ulcorner\top\urcorner=$ true, $\ulcorner\perp\urcorner=$ false, $\ulcorner 0\urcorner=0,\ulcorner 1\urcorner=1, \ldots,\ulcorner n\urcorner=n$ for $n \in \mathbb{N}$, and $\ulcorner x\urcorner=x$ for $x \in \mathcal{X}$.

Among the set of programs of PIL, we consider the subset of programs whose evaluation (under assumption A) always terminates in accept or refuse, whatever the input is. Those programs are called well-formed programs. 


$$
\begin{aligned}
& \overline{\Gamma, \Delta \vdash\ulcorner b\urcorner: w f(b \in \mathbb{B})} \quad \frac{\Gamma, \Delta \vdash \mathrm{t}_{1}: w f \quad \Gamma, \Delta \vdash \mathrm{t}_{2}: w f}{\Gamma, \Delta \vdash \mathrm{eq}\left(\mathrm{t}_{1}, \mathrm{t}_{2}\right): w f} \\
& \frac{\Gamma, \Delta \vdash \mathrm{t}: w f}{\Gamma, \Delta \vdash\ulcorner t\urcorner: w f(t \in \mathcal{T}(\mathcal{F}))} \quad \frac{\Gamma, \Delta \operatorname{subterm}_{f}(\mathrm{t}, i): w f}{\Gamma, \Delta f}(\mathrm{t}, f) \in \Delta \text { and } i \in[1 . . \operatorname{ar}(f)] \\
& \overline{\Gamma, \Delta \vdash \text { accept }: w f} \quad \frac{\Gamma, \Delta \vdash \mathrm{t}: w f \quad \Gamma:: v, \Delta \vdash i: w f}{\Gamma, \Delta \vdash \operatorname{let}(v, \mathrm{t}, i): w f} \\
& \overline{\Gamma, \Delta \vdash \text { refuse }: w f} \\
& \frac{\Gamma, \Delta \vdash \text { is_fsym }(\mathrm{t},\ulcorner f\urcorner): w f \quad \Gamma, \Delta::(\mathrm{t},\ulcorner f\urcorner) \vdash i_{1}: w f \quad \Gamma, \Delta \vdash i_{2}: w f}{\Gamma, \Delta \vdash \operatorname{if}\left(\text { is_fsym }(\mathrm{t},\ulcorner f\urcorner), i_{1}, i_{2}\right): w f} \\
& \frac{\Gamma, \Delta \vdash e: w f \quad \Gamma, \Delta \vdash i_{1}: w f \quad \Gamma, \Delta \vdash i_{2}: w f}{\Gamma, \Delta \vdash \operatorname{if}\left(e, i_{1}, i_{2}\right): w f} \text { if } e \neq \text { is_fsym }(\mathrm{t},\ulcorner f\urcorner)
\end{aligned}
$$

Figure 2: Type system for checking validity

Definition 3 A program $\pi \in$ PIL is said to be well-formed when it satisfies the following properties.

- Each expression $\operatorname{subterm}_{f}(t, n)$ is such that $t$ belongs to $\langle$ term $\rangle$, is_f $\left.\operatorname{sym}(t,\ulcorner f\urcorner)\right) \equiv$ true and $n \in[1 . . \operatorname{ar}(f)]$.

(In practice, we verify that each expression of the form $\operatorname{subterm}_{f}(t, n)$ belongs to the then part of an instruction if $($ is_f $f \operatorname{sym}(t,\ulcorner f\urcorner), \ldots)$.)

- Each variable appearing in a sub-expression is previously initialized by a let construct, or in the evaluation environment.

We introduce here a simple type system for verifying that a given program is well-formed, in a particular context (modeling the evaluation environment). This context is formed by the variables which have been introduced in the evaluation environment, noted $\Gamma$, and a list of couples $(\langle t e r m\rangle$, symbol), noted $\Delta$, representing the fact that in the evaluation environments, the root symbol of a given term is known.

Property 1 A PIL-program $\pi$ is said well-formed in an evaluation environment if and only if we can build a derivation of $\Gamma, \Delta \vdash \pi: w f$ in the type system presented in Figure Q 2 . $\Gamma$ contains the variables initialized by the environment, and $\Delta$ stores which terms have a particular root symbol.

Proof 1 Let $\pi$ a PIL-program, $\Gamma, \Delta$ contexts such that there is a derivation $\Gamma, \Delta \vdash \pi: w f$ in the type system of Figure Q.

So for each variable $v$ in $\pi$, it exists contexts $\Gamma^{\prime}, \Delta^{\prime}$ such that $\Gamma^{\prime}, \Delta^{\prime} \vdash v: w f$, and thus $v \in \Gamma^{\prime}$. Since $v$ can only be introduced in the context $\Gamma^{\prime}$ either by early initialisation, or by applying the typing rule for let, the variable $v$ has been initialized. Also, for each $\operatorname{subterm}_{f}(t, i)$ construct in $\pi$, it exists contexts $\Gamma^{\prime}, \Delta^{\prime}$ such that $\Gamma^{\prime}, \Delta^{\prime} \vdash \operatorname{subterm}_{f}(t, i): w f$, and $(t,\ulcorner f\urcorner) \in \Delta^{\prime}$. Since $(t,\ulcorner f\urcorner)$ can only be introduced in the context $\Delta^{\prime}$ by applying the typing rule for $\operatorname{if}\left(\operatorname{is} \_f_{\operatorname{sym}}(t,\ulcorner f\urcorner), i_{1}, i_{2}\right)$ or by a previous test, the representation $t$ has been checked to have root symbol $f$.

Let $\pi$ a well-formed PIL-program in an evaluation environment. If we initialize the contexts $\Gamma$ and $\Delta$ with the variables already instantiated in the environment, and with which terms have a particular root symbol, we can build a derivation of $\Gamma, \Delta \vdash \pi: w f$, since the typing rules for variables and subterm contructs will apply, each variable being either instantiated in the initial environment, or introduced by a let construct before its use, and root symbol of terms and arities being checked before the use of a subterm construct, either with a test in the program or in the evaluation environment.

In practice, when verifying that a given program is well-formed, we initialize the environments with the set of input variables of the program as $\Gamma$ (corresponding to the subject against which the program matches), and an empty list of couples $\Delta$. 
Notice that the well-formedness of a PIL-program is linearly decidable, since this property can be decided by the type system in Figure 2

The program given in Example 3 is well-formed in the environment $\Gamma=\{s\}, \Delta=\emptyset$, since subterm $_{f}(\ulcorner s\urcorner,\ulcorner 1\urcorner)$ is protected by the construct if $\left(\operatorname{is}_{\mathbf{S}} \mathbf{f} \operatorname{sym}(\ulcorner s\urcorner,\ulcorner f\urcorner), \ldots\right)$ with $1 \in[1 . . \operatorname{ar}(f)],\ulcorner x\urcorner$ is introduced by a let, and $\ulcorner s\urcorner$ is in $\Gamma$.

On the contrary, the program: if (is_f $\operatorname{sym}(\ulcorner s\urcorner,\ulcorner f\urcorner)$, if ( eq $\left(\ulcorner x\urcorner, \operatorname{subterm}_{g}(\ulcorner s\urcorner,\ulcorner 1\urcorner)\right)$, accept, refuse), refuse) is not well-formed in the same environment for two reasons: $\ulcorner x\urcorner$ is not introduced by a let, and subterm $_{g}$ is not guarded by an if (is_f $\operatorname{sym}(\ulcorner s\urcorner,\ulcorner g\urcorner), \ldots)$.

\subsection{Environments}

Given a matching problem, its satisfiability is of course of interest. But in most applications it is not enough and we need to compute a witness: i.e. a substitution which assigns values to the variables of the pattern. In this section, we introduce the notion of environment, which models the memory of a program during its evaluation. To represent a substitution, we model an environment by a stack of assignments of concrete terms to variable names. In addition, we also define a function $\Phi$ which goes back from environments to algebraic substitutions.

Definition 4 An atomic environment $\epsilon$ is an assignment from $\ulcorner\mathcal{X}\urcorner$ to $\ulcorner\mathcal{T}(\mathcal{F})\urcorner$, written $[x \leftarrow\ulcorner t\urcorner]$. The composition of environments is left-associative, and written $\left[x_{1} \leftarrow\left\ulcorner t_{1}\right\urcorner\right]\left[x_{2} \leftarrow\left\ulcorner t_{2}\right\urcorner\right] \cdots\left[x_{k} \leftarrow\left\ulcorner t_{k}\right\urcorner\right]$. Its application is such that:

$$
\epsilon[x \leftarrow\ulcorner t\urcorner](y)= \begin{cases}\ulcorner t\urcorner & \text { if } y \equiv x \\ \epsilon(y) & \text { otherwise }\end{cases}
$$

We extend the notion of environment to a morphism $\epsilon^{\prime}$ from PIL to PIL, and we note $\mathcal{E} n v$ the set of all environments.

Definition 5 Given $\mathcal{F}$ and $\mathcal{X}$, we define the mapping $\Phi$ from environments to substitutions, by $\Phi(\epsilon)=\sigma$ where:

$$
\sigma=\left\{x_{i} \mapsto t_{i} \mid \epsilon\left(\left\ulcorner x_{i}\right\urcorner\right)=\left\ulcorner t_{i}\right\urcorner \text { with } x_{i} \in \mathcal{X} \text { and } t_{i} \in \mathcal{T}(\mathcal{F})\right\}
$$

Hence, to prove the correctness of the compiled code $\pi_{p}$, we want to ensure that, for a given model of evaluation "eval" and for each term $t$, the following diagram commutes:

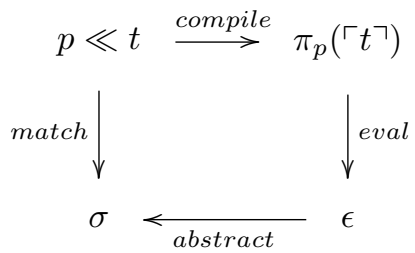

We are now going to make the evaluation mechanism explicit.

\subsection{Big-step semantics}

We use a big step semantics à la Kahn [?] to express the behavior of the PIL evaluation mechanism. The reduction relation of this big-step semantics is expressed on couples made of an environment and an instruction, denoted $\langle\epsilon, i\rangle$. The reduction relation for the big-step semantics is:

$$
\langle\epsilon, i\rangle \mapsto_{b s}\left\langle\epsilon^{\prime}, i^{\prime}\right\rangle \text {, with } i, i^{\prime} \in\langle i n s t r\rangle \text {, and } \epsilon, \epsilon^{\prime} \in \mathcal{E} n v
$$

and the rules for the big-step semantics are presented in Figure 3 . The presented semantics is quite standard, however, the reader should note that conditions are evaluated modulo a formal anchor \ulcorner\urcorner and the equivalences given in Section 2.3. In the line of Example 3, if we evaluate the program in the environment where $s$ is bound to $\ulcorner f(a)\urcorner$, the condition $[s \leftarrow\ulcorner f(a)\urcorner]($ is_f $\operatorname{sym}(s,\ulcorner f\urcorner)) \equiv$ true is equivalent to the condition $\ulcorner\mathcal{S y m b}(f(a))=f\urcorner \equiv$ true, which in this case is true since the top symbol of $f(a)$ is $f$. 


$$
\begin{array}{lr}
\overline{\langle\epsilon, \text { accept }\rangle \mapsto_{b s}\langle\epsilon, \text { accept }\rangle} & \begin{array}{c}
\text { (accept) } \\
\text { (refuse) }
\end{array} \\
\frac{\langle\epsilon, \text { refuse }\rangle \mapsto_{b s}\langle\epsilon, \text { refuse }\rangle}{\left\langle\epsilon, i_{1}\right\rangle \mapsto_{b s}\left\langle\epsilon^{\prime}, i\right\rangle} & \\
\frac{\left\langle\epsilon, i_{2}\right\rangle \mapsto_{b s}\left\langle\epsilon^{\prime}, i\right\rangle}{\left.\left\langle\epsilon, i_{1}, i_{2}\right)\right\rangle \mapsto_{b s}\left\langle\epsilon^{\prime}, i\right\rangle} \text { if } \epsilon(e) \equiv \text { true } & \text { (iftrue) } \\
\frac{\left\langle\epsilon, \text { if }\left(e, i_{1}, i_{2}\right)\right\rangle \mapsto_{b s}\left\langle\epsilon^{\prime}, i\right\rangle}{} \text { if } \epsilon(e) \equiv \text { false } & \text { (iffalse) } \\
\frac{\left\langle\epsilon\left[x \leftarrow\ulcorner t], i_{1}\right\rangle \mapsto_{b s}\left\langle\epsilon^{\prime}, i\right\rangle\right.}{\left\langle\epsilon, \operatorname{let}\left(x, u, i_{1}\right)\right\rangle \mapsto_{b s}\left\langle\epsilon^{\prime}, i\right\rangle} \text { if } \epsilon(u) \equiv\ulcorner t\urcorner & \text { (let) }
\end{array}
$$

Figure 3: Big-step semantics for PIL

\section{Certified compilation}

Given a PIL program $\pi$ and a pattern $p$, we first define what means for $\pi$ to be a correct compilation of $p$. Intuitively, this asserts that the execution of $\pi_{p}$ will go into the accept state only if the pattern $p$ matches $t$. Conversely, when $p$ does not match $t$, the program shall go into the refuse state.

Then, we state the correctness theorem and properties that show how the presented approach can be used to formally certify that a matching problem is correctly compiled. This result will be extended in Section 5 to match constructs as seen in Caml or Tom, where a subject is matched against multiple patterns.

\subsection{Pattern-matching compilation correctness}

The big-step semantics introduced previously allows us to define now the notion of correct compilation $\pi_{p}$ of a pattern $p \in \mathcal{T}(\mathcal{F}, \mathcal{X})$.

Definition 6 Given a formal anchor \ulcorner\urcorner , a well-formed program $\pi_{p}$ is a sound compilation of $p$ when both:

$$
\begin{aligned}
\forall \epsilon, \epsilon^{\prime} \in \mathcal{E} n v, \forall t \in \mathcal{T}(\mathcal{F}), & \\
\left\langle\epsilon, \pi_{p}(\ulcorner t\urcorner)\right\rangle \mapsto_{b s}\left\langle\epsilon^{\prime}, \text { accept }\right\rangle \Rightarrow & \Phi\left(\epsilon^{\prime}\right)(p)=t \\
& \left(\operatorname{sound}_{O K}\right) \\
\left\langle\epsilon, \pi_{p}(\ulcorner t\urcorner)\right\rangle \mapsto_{b s}\left\langle\epsilon^{\prime}, \text { refuse }\right\rangle \Rightarrow & p \nless t \\
& \left(\text { sound }_{K O}\right)
\end{aligned}
$$

Definition 7 Given a formal anchor \ulcorner\urcorner , a well-formed program $\pi_{p}$ is a complete compilation of $p$ when both:

$$
\begin{aligned}
& \forall \epsilon \in \mathcal{E} n v, \forall t \in \mathcal{T}(\mathcal{F}) \\
& p \ll t \Rightarrow \quad \exists \epsilon^{\prime} \in \mathcal{E} n v,\left\langle\epsilon, \pi_{p}(\ulcorner t\urcorner)\right\rangle \mapsto_{b s}\left\langle\epsilon^{\prime} \text {, accept }\right\rangle \\
& \wedge \Phi\left(\epsilon^{\prime}\right)(p)=t \quad\left(\text { complete }_{O K}\right) \\
& p \nless t \Rightarrow \quad \exists \epsilon^{\prime} \in \mathcal{E} n v,\left\langle\epsilon, \pi_{p}(\ulcorner t\urcorner)\right\rangle \mapsto b s\left\langle\epsilon^{\prime}, \text { refuse }\right\rangle \\
& \text { ( } \left.\text { complete }_{K O}\right)
\end{aligned}
$$

Definition 8 A compilation of a pattern $p$ into a program $\pi_{p}$ is said correct, when it is sound and complete.

Informally, Definition 8 says that a program $\pi_{p}$ is a correct compilation of $p$ when its execution of $\pi_{p}$ leads to accept for all subjects which are matched by $p$, and reciprocally. The execution should also lead to refuse if and only if $p$ does not match the subject. In addition, Definition 6 and 7 ensure that the environment $\epsilon^{\prime}$ computed by the execution of $\pi_{p}$ corresponds to a substitution $\sigma$ such that $\sigma(p)=t$.

In order to certify that a given program $\pi_{p}$ corresponds to a correct compilation of a pattern $p$, Theorem 1 shows that it is sufficient to compute all derivations of $\pi_{p}$ to know whether the compilation is correct or not. 
Theorem 1 Given a formal anchor $\left\ulcorner\right.$, a pattern $p \in \mathcal{T}(\mathcal{F}, \mathcal{X})$, and a well-formed program $\pi_{p} \in$ PIL, we have:

$$
\begin{gathered}
\pi_{p} \text { is a correct compilation of } p \\
\Longleftrightarrow \\
\forall \epsilon, \epsilon^{\prime} \in \mathcal{E} n v, \forall t \in \mathcal{T}(\mathcal{F}), \\
\left\langle\epsilon, \pi_{p}(\ulcorner t\urcorner)\right\rangle \mapsto b s\left\langle\epsilon^{\prime}, \text { accept }\right\rangle \Leftrightarrow \Phi\left(\epsilon^{\prime}\right)(p)=t
\end{gathered}
$$

Proof 2 By application of Properties 2 and 3 below.

Property 2 For all environments $\epsilon \in \mathcal{E} n v$, the derivation of a well-formed instruction $i \in\langle$ instr $\rangle$ in the environment $\Gamma, \Delta$ leads trivially to accept or to refuse, and the reduction is unique.

Proof 3 We proceed by induction over the structure of the instruction $i$.

We take as induction hypothesis that for all environments $\epsilon \in \mathcal{E} n v$, the derivation of a well-formed instruction $i \in\langle$ instr $\rangle$ in the environment $\Gamma, \Delta$ leads to accept or to refuse, and the reduction is unique.

- when $i=$ accept (resp. $i=$ refuse), only one inference rule can be applied: the axiom (accept) (resp. (refuse)). So the derivation leads uniquely either to accept or refuse.

- when $i=\operatorname{let}\left(x, u, i_{1}\right)$, only one inference rule can be applied: the (let) rule:

$$
\frac{\left\langle\epsilon[x \leftarrow\ulcorner t\urcorner], i_{1}\right\rangle \mapsto_{b s}\left\langle\epsilon^{\prime}, i_{2}\right\rangle}{\left\langle\epsilon, \operatorname{let}\left(x, u, i_{1}\right)\right\rangle \mapsto_{b s}\left\langle\epsilon^{\prime}, i_{2}\right\rangle} \text { if } \epsilon(u) \equiv\ulcorner t\urcorner
$$

To complete the proof, we have to show that $\exists t \in \mathcal{T}(\mathcal{F})$ such that $\epsilon(u) \equiv\ulcorner t\urcorner$. We know that $i$ is a well-formed instruction in the context $\Gamma, \Delta$, so each variable occurring in $u$ is previously initialized:

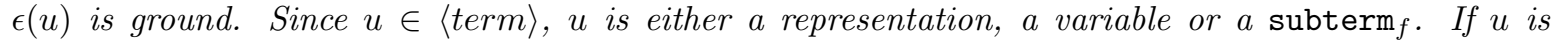
already a term representation, there is no problem. If $u$ is a variable, $u$ has been instantiated by term representation in the evaluation environment, since it is well-formed. The well-formed-ness of $i$ ensures that each subterm $_{f}$ construct is encapsulated by an if (is_f $\left.\operatorname{sym}(\ulcorner\ldots\urcorner,\ulcorner f\urcorner), \ldots\right)$ and that each variable is initialized. Also, all subterm $_{f}$ expressions are $\equiv$-equivalent (see Definition 2) to a term representation: $\exists t \in \mathcal{T}(\mathcal{F})$ such that $\epsilon(u) \equiv\ulcorner t\urcorner$.

By induction hypothesis, we know that the derivation of $i_{1}$ leads either to accept or refuse in a unique way, so the derivation of $i$ is also unique and leads either to accept or refuse.

- when $i=\operatorname{if}\left(e, i_{1}, i_{2}\right)$, using similar arguments, we show that each 〈term〉 occurring in e is $\equiv$-equivalent to a term representation. Since the expression e is either a boolean representation, an is_fsym, or an eq (where subterms are term representations), e is by definition $\equiv$-equivalent to the representation of a boolean. Thus, we have either $e \equiv$ true or $e \equiv$ false.

When $e \equiv$ true (resp. $e$ E false), the only applicable rule is (iftrue) (resp. (iffalse)), and we have:

$$
\frac{\left\langle\epsilon, i_{1}\right\rangle \mapsto_{b s}\left\langle\epsilon^{\prime}, i_{3}\right\rangle}{\left\langle\epsilon, \text { if }\left(e, i_{1}, i_{2}\right)\right\rangle \mapsto_{b s}\left\langle\epsilon^{\prime}, i_{3}\right\rangle} \text { if } \epsilon(e) \equiv \text { true } \quad \text { (iftrue) }
$$

by induction hypothesis the reduction of $i_{1}$ (resp. $i_{2}$ ) in the environment $\epsilon$ leads either to accept or refuse in an unique way, so the reduction of $i$ does the same.

Thus, given $\epsilon \in \mathcal{E} n v$, the reduction of a well-formed instruction $i$ in an environment $\Gamma, \Delta$ leads either to accept or to refuse, and the reduction is unique.

Property 3 Given a formal anchor \ulcorner\urcorner and a well-formed program $\pi_{p} \in$ PIL, we have:

$$
\forall \epsilon \in \mathcal{E} n v, \forall t \in \mathcal{T}(\mathcal{F}),\left(\text { sound }_{O K}\right) \Rightarrow\left(\text { complete }_{K O}\right) \text { and }\left(\text { complete }_{O K}\right) \Rightarrow\left(\text { sound }_{K O}\right)
$$


Proof 4 Let us suppose (sound $\left.{ }_{O K}\right)$ and $p \nless t$. Since the derivation of $\left\langle\epsilon, \pi_{p}(\ulcorner t\urcorner)\right\rangle$ is unique (Property 2), we cannot have $\left\langle\epsilon, \pi_{p}(\ulcorner t\urcorner)\right\rangle \mapsto_{b s}\left\langle\epsilon^{\prime}\right.$, accept $\rangle$ without contradicting $p \nless t$. Property 2 says also that a derivation either leads to accept or refuse. Thus, we necessarily have $\left\langle\epsilon, \pi_{p}(\ulcorner t\urcorner)\right\rangle \mapsto b s\left\langle\epsilon^{\prime}\right.$, refuse $\rangle$, and thus $\left(\right.$ sound $\left._{O K}\right) \Rightarrow\left(\right.$ complete $\left._{K O}\right)$.

Let us now suppose (complete $\left.{ }_{O K}\right)$ and that $\exists \epsilon^{\prime} \in \mathcal{E} n v,\left\langle\epsilon, \pi_{p}(\ulcorner t\urcorner)\right\rangle \mapsto_{b s}\left\langle\epsilon^{\prime}\right.$, refuse $\rangle$. We have to show that $p \ll t$. If $p \ll t$, then by (complete $\left.{ }_{O K}\right)$ we have $\exists \epsilon^{\prime} \in \mathcal{E} n v,\left\langle\epsilon, \pi_{p}(\ulcorner t\urcorner)\right\rangle \mapsto b s\left\langle\epsilon^{\prime}\right.$, accept $\rangle$. This is in contradiction with the uniqueness of the derivation of $\left\langle\epsilon, \pi_{p}\right\rangle$, so we have $p \nless t$. Hence (complete $\left.e_{\mathrm{OK}}\right) \Rightarrow$ $\left(\right.$ sound $\left._{K O}\right)$.

\subsection{Interpreting the big-step semantics}

Theorem 1 is the key result to prove that a program $\pi_{p}$ is correct. However, the equivalence between $\left\langle\epsilon, \pi_{p}(\ulcorner t\urcorner)\right\rangle \mapsto_{b s}\left\langle\epsilon^{\prime}\right.$, accept $\rangle$ and $\Phi\left(\epsilon^{\prime}\right)(p)=t$ is difficult to prove since the big-step semantics has to be modeled and used in the proof. To solve this problem, we use a very simple form of abstract interpretation (because the symbolic simulation can be done without approximation) to statically derive a set of constraints characterizing the program behavior in the spirit of [?, ?]. Therefore, given a program $\pi_{p}$, we compute a set of constraints $\mathcal{C} \pi_{p}$ such that, to prove a program correct, we show that for all $t$, " $t$ satisfies $\mathcal{C} \pi_{p}$ " if and only if "there exists $\epsilon$ such that $\Phi(\epsilon)(p)=t$ ".

In practice, this result is useful because the big-step semantics $\mapsto_{b s}$ does not appear anymore explicitly. This makes the proof smaller, and easier to handle by an automatic theorem prover.

Definition 9 A big-step derivation leading to accept is called successful. Let $\mathbf{s}$ be an input variable and $\pi_{p}$ be a PIL well-formed program. To each successful big-step derivation $\mathcal{D}$ we associate the conjunction $\mathcal{C}_{\mathcal{D}}$ of all constraints raised by the derivation. $\mathcal{C}_{p}(\mathbf{s})$ is defined as the disjunction of all constraints $\mathcal{C}_{\mathcal{D}}$ for all successful big-step derivations.

In practice, we can use a dedicated tool to extract the constraints from a program. Starting from an environment $\epsilon$ containing only the input variable, it is sufficient to compute all big-step derivations leading to accept: $\left\langle\epsilon, \pi_{p}(\ulcorner t\urcorner)\right\rangle \mapsto_{b s}\left\langle\epsilon^{\prime}\right.$, accept $\rangle$. The constraints corresponds to the conditions raised by the application of a big-step rule, given Figure 3 . Let us note that the number of generated constraints is linear in the size of the program. In practice, for a single pattern, the program is usually linear in the size of the pattern.

Given a term $t$, we note $\mathcal{C} \pi_{p}(t)$ the fact that $t$ satisfies the constraint $\mathcal{C} \pi_{p}$. An example of such a constraint is given in Figure 5

Property 4 Given a formal anchor $\left\ulcorner\right.$, and a well-formed program $\pi_{p} \in \mathrm{PIL}$, we have:

$$
\begin{gathered}
\forall \epsilon \in \mathcal{E} n v, \forall t \in \mathcal{T}(\mathcal{F}), \\
\exists \epsilon^{\prime} \in \mathcal{E} n v,\left\langle\epsilon, \pi_{p}(\ulcorner t\urcorner)\right\rangle \mapsto b s\left\langle\epsilon^{\prime}, \text { accept }\right\rangle \Leftrightarrow \mathcal{C} \pi_{p}(t)
\end{gathered}
$$

Proof 5 It is clear that if the derivation $\left\langle\epsilon, \pi_{p}(\ulcorner t\urcorner)\right\rangle \mapsto_{b s}\left\langle\epsilon^{\prime}\right.$, accept $\rangle$ is possible, then $t$ satisfies the constraint $\mathcal{C} \pi_{p}$. On the other hand, if $t$ satisfies the constraint $\mathcal{C} \pi_{p}$, then a derivation leading to accept can be built.

Theorem 2 Given a formal anchor $\left\ulcorner\right.$, a pattern $p \in \mathcal{T}(\mathcal{F}, \mathcal{X})$, and a well-formed program $\pi_{p} \in \mathrm{PIL}$, we have:

$$
\pi_{p} \text { is a correct compilation of } p
$$

$$
\forall t \in \mathcal{T}(\mathcal{F}), \mathcal{C} \pi_{p}(t) \Leftrightarrow \exists \epsilon^{\prime} \in \mathcal{E} n v, \Phi\left(\epsilon^{\prime}\right)(p)=t
$$

This theorem can be used to prove correct the compilation of a pattern. As illustrated by Figure 4. given a pattern $p$, a condition over a term $t$ written $\mathcal{C} p, \sigma$ can be extracted. In general this condition is of the form $\exists \sigma, \sigma(p)=t$, but, by using a matching algorithm, the substitution $\sigma$ can be instantiated by $\left\{x_{1} \mapsto t_{1}, \ldots, x_{k} \mapsto t_{k}\right\}$, where $t_{1}, \ldots, t_{k}$ correspond to subterms of a subject $t$. When satisfied, this condition ensures that $p$ matches $t$. 


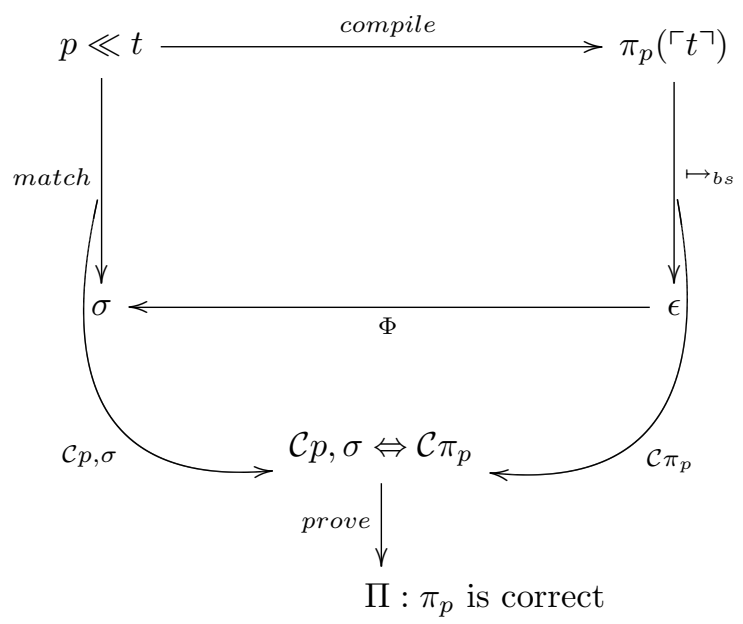

Figure 4: General schema of certification

Similarly, given a program $\pi_{p}$, the constraint $\mathcal{C} \pi_{p}$ can be computed. By application of Theorem 4 we know that if we can prove the equivalence between these two conditions, then the program $\pi_{p}$ is a correct compilation of $p$. This proof can be done by an automatic prover to provide a formal proof $\Pi$.

\subsection{Working example}

As an example, let us consider the pattern $g(x, b)$, with $x \in \mathcal{X}$. Let us now suppose that our compiler produces the following program, where $\mathrm{s}$ is an input variable:

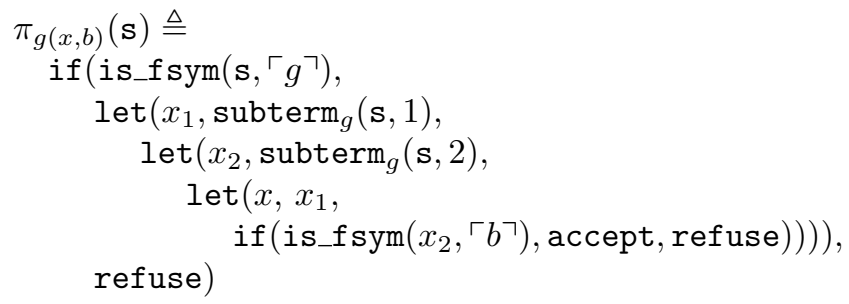

Given a term $t$ and an environment $\epsilon_{0}=[\mathbf{s} \leftarrow\ulcorner t\urcorner]$, let us suppose that $\left\langle\epsilon_{0}, \pi_{g(x, b)}(\mathbf{s})\right\rangle \mapsto b s\left\langle\epsilon^{\prime}\right.$, accept $\rangle$. Figure 5 shows the unique derivation that can be computed by applying the inference rules defined in Section 3.3 .

To make this derivation possible, the following set of constraints has to be satisfied:

$$
\mathcal{C} \pi_{p}(\mathbf{s})=\left\{\begin{array}{lll}
\epsilon_{0}(\text { is_fsym }(\mathbf{s},\ulcorner g\urcorner)) & \equiv \text { true } & (1) \\
\epsilon_{0}\left(\operatorname{subterm}_{g}(\mathbf{s}, 1)\right) & \equiv\left\ulcorner t_{\mid 1}\right\urcorner(2) \\
\epsilon_{1}\left(\operatorname{subterm}_{g}(\mathbf{s}, 2)\right) & \equiv\left\ulcorner t_{\mid 2}\right\urcorner(3) \\
\epsilon_{2}\left(x_{1}\right) & \equiv\left\ulcorner t_{\mid 1}\right\urcorner(4) \\
\epsilon_{3}\left(\text { is_f } \operatorname{sym}\left(x_{2},\ulcorner b\urcorner\right)\right) & \equiv \text { true }
\end{array}\right.
$$

(1) and (5) can be simplified using the equations of the formal anchor, (2), (3), and (4) are tautologies. Thus, to prove the correctness of $\pi_{p}$, we have to prove the equivalence:

$$
\begin{aligned}
\forall t & \in \mathcal{T}(\mathcal{F}), \\
\sigma(g(x, b))=t & \wedge \sigma=\left\{x \mapsto t_{\mid 1}\right\} \\
& \Longleftrightarrow \\
\mathcal{S} y m b(t)=g & \wedge \mathcal{S} y m b\left(t_{\mid 2}\right)=b
\end{aligned}
$$




$$
\begin{aligned}
& \left.\operatorname{Let}_{3} \triangleq \operatorname{let}\left(x, x_{1} \text {, if(is_fsym }\left(x_{2},\ulcorner b\urcorner\right) \text {, accept, refuse }\right)\right) \mid \epsilon_{0}=[\mathbf{s} \leftarrow\ulcorner t\urcorner] \\
& \operatorname{Let}_{2} \triangleq \operatorname{let}\left(x_{2}, \operatorname{subterm}_{g}(\mathrm{~s}, 2), \operatorname{Let}_{3}\right) \quad \epsilon_{1}=\epsilon_{0}\left[x_{1} \leftarrow\left\ulcorner t_{\mid 1}\right\urcorner\right] \\
& \begin{array}{l|l}
\operatorname{Let}_{1} \triangleq \operatorname{let}\left(x_{1}, \operatorname{subterm}_{g}(\mathbf{s}, 1), \operatorname{Let}_{2}\right) & \epsilon_{2}=\epsilon_{1}\left[x_{2} \leftarrow\left\ulcorner t_{\mid 2}\right\urcorner\right] \\
\epsilon_{3}=\epsilon_{2}\left[x \leftarrow\left\ulcorner t_{11}\right\urcorner\right]
\end{array}
\end{aligned}
$$

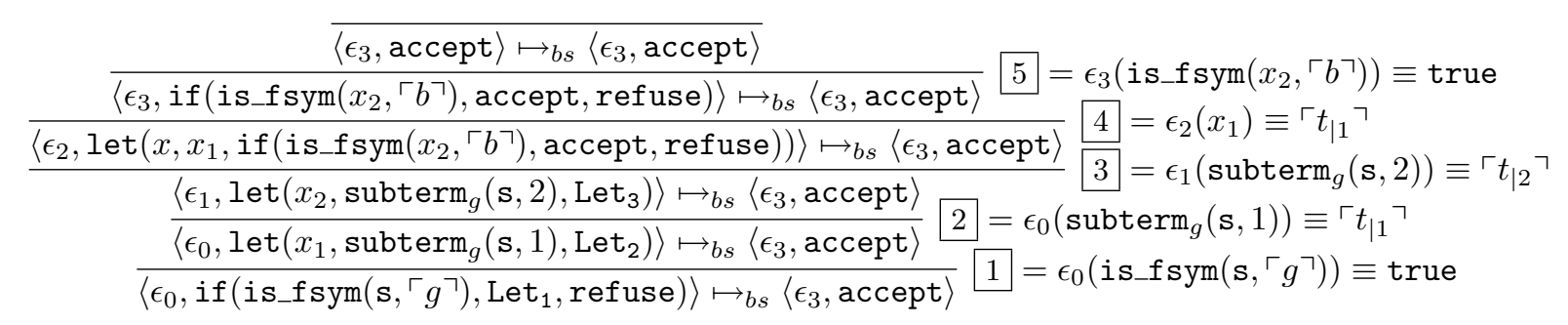

Figure 5: Example of derivation leading to accept. We have $\mathcal{C} \pi_{p}(\mathbf{s})=\{1 \wedge 2 \wedge 3 \wedge 4 \wedge 5\}$

This is proved by first applying the substitution in the first part of the proof obligation, and then using the definitions of terms, symbols and subterms.

For this example, with $g$ a function symbol of arity 2 and $b$ a constant symbol, the mapping definition leads to the following axioms:

$$
\begin{aligned}
& \forall t \in \mathcal{T}(\mathcal{F}), \operatorname{Symb}(t)=g \Leftrightarrow \exists x, y \in \mathcal{T}(\mathcal{F}), t=g(x, y) \\
& \forall t \in \mathcal{T}(\mathcal{F}), \mathcal{S} y m b(t)=b \Leftrightarrow(t=b) \\
& \forall x, y \in \mathcal{T}(\mathcal{F}), g(x, y)_{\mid 1}=x \\
& \forall x, y \in \mathcal{T}(\mathcal{F}), g(x, y)_{\mid 2}=y
\end{aligned}
$$

The first two axioms define the meaning of the $\mathcal{S} y m b$ function. The two remaining axioms define the subterm function over terms rooted by the symbol $g$. As we use a first order prover, we need such a definition for each symbol function and for each subterm.

To prove the left to right implication, we simply apply the substitution to the left part, and then apply the first axiom, to obtain $\mathcal{S} y m b(t)=g$, and the fourth axiom to obtain $\mathcal{S} y m b\left(t_{\mid 2}\right)=b$.

To prove the remaining implication $(\Leftarrow)$, we apply the first axiom to the first constraint, obtaining $\exists x, y$ such that $t=g(x, y)$. We then apply the third and fourth axiom, to instantiate $x$ and $y$ by $t_{\mid 1}$ and $t_{\mid 2}$. The second constraint with the second axiom gives $t_{\mid 2}=b$. We can then obtain $g\left(t_{\mid 1}, b\right)$, and extract the substitution.

The form of such propositions is rather simple but a huge number of them could be generated, so this kind of proof should better be done by an automated theorem prover. In our implementation, we are using Zenon [?], a first order tableau based automatic theorem prover. One of the nice capability of Zenon is to generate a formal proof in Coq when a theorem can be proved. In our case, a witness of correctness is generated and associated to the generated code.

Another proof approach will be to use theorem proving modulo [?] using in particular the axioms issued from the mapping definition.

\section{Extension to match constructs}

Our method can be extended to support match constructs à la ML, Caml or Tom. We consider not only single patterns, but also constructs of the form match $s$ with $\left(p_{1} \rightarrow a_{1}\right), \ldots,\left(p_{n} \rightarrow a_{n}\right)$. The semantics of this construct is the following: if $p_{1}$ matches the subject $s$, the program goes into the accept state, 
and to keep track of the pattern number, the accept state is labeled by $p_{1}$, noted accept $p_{1}$. Otherwise, the subproblem match $s$ with $\left(p_{2} \rightarrow a_{2}\right), \ldots,\left(p_{n} \rightarrow a_{n}\right)$ is considered. When no pattern $p_{i}$ matches $s$, the program goes into the refuse state.

This new match construct can be easily compiled using the intermediate language PIL. However, to avoid code duplication and to ease expression in PIL, it is useful to consider the sequence construct: $\langle$ instr $\rangle ;\langle$ instr $\rangle$.

This sequence construct has the following big-step semantics:

$$
\begin{array}{ll}
\frac{\left\langle\epsilon, i_{1}\right\rangle \mapsto_{b s}\left\langle\epsilon^{\prime}, \text { accept }_{p}\right\rangle}{\left\langle\epsilon, i_{1} ; i_{2}\right\rangle \mapsto_{b s}\left\langle\epsilon^{\prime}, \text { accept }_{p}\right\rangle} & \left(\text { seq }_{a}\right) \\
\frac{\left\langle\epsilon, i_{1}\right\rangle \mapsto_{b s}\left\langle\epsilon^{\prime}, \text { refuse }\right\rangle \quad\left\langle\epsilon^{\prime}, i_{2}\right\rangle \mapsto_{b s}\left\langle\epsilon^{\prime \prime}, i\right\rangle}{\left\langle\epsilon, i_{1} ; i_{2}\right\rangle \mapsto_{b s}\left\langle\epsilon^{\prime \prime}, i\right\rangle} \quad\left(\text { seq }_{b}\right)
\end{array}
$$

It is easy to show that adding the sequence rules does not break the property of uniqueness for the derivation of a well-formed instruction. The notion of correct compilation of a match construct is an extension of the definition of the correct compilation of a pattern. The difference comes from the presence of multiple patterns. Hence, when a pattern is selected to fire a rule (accept ${ }_{p}$ in our terminology), we should ensure that all previous patterns do not match the subject. In the following, we do not make any assumptions on the form of the code to validate. This ensures that we can consider any optimizations of the matching code, like factorization of common tests.

Let $\mathcal{P}_{m}$ be the set of patterns for the match construct, and $<$ a total ordering relation for patterns in $\mathcal{P}_{m}$. In the case of $\mathrm{ML}$ for example, we define $<$ by the textual ordering: $p_{i}<p_{j}$ if $p_{i}$ occurs before $p_{j}$ in the match construct.

Definition 10 Given a formal anchor $\left\ulcorner\right.$, a well-formed program $\pi_{m}$ is a sound compilation of $m$ when both:

$$
\begin{aligned}
& \forall \epsilon \in \mathcal{E} n v, \forall t \in \mathcal{T}(\mathcal{F}): \\
& \forall p \in \mathcal{P}_{m}, \exists \epsilon^{\prime} \in \mathcal{E} n v,\left\langle\epsilon, \pi_{m}(\ulcorner t\urcorner)\right\rangle \mapsto_{b s}\left\langle\epsilon^{\prime}, \text { accept }_{p}\right\rangle \\
& \Rightarrow \Phi\left(\epsilon^{\prime}\right)(p)=t \wedge\left(\forall p^{\prime} \in \mathcal{P}_{m} \text { s.t. } p^{\prime}<p, \Phi\left(\epsilon^{\prime}\right)\left(p^{\prime}\right) \neq t\right) \\
& \quad\left(M \text { sound }_{O K}\right) \\
& \exists \epsilon^{\prime} \in \mathcal{E} n v,\left\langle\epsilon, \pi_{m}(\ulcorner t\urcorner)\right\rangle \mapsto_{b s}\left\langle\epsilon^{\prime}, \text { refuse }\right\rangle \Rightarrow \forall p \in \mathcal{P}_{m}, p \nless t
\end{aligned}
$$

$\left(M \operatorname{sound}_{K O}\right)$

Definition 11 Given a formal anchor $\left\ulcorner\right.$, a well-formed program $\pi_{m}$ is a complete compilation of $m$ when both:

$$
\begin{aligned}
& \forall \epsilon \in \mathcal{E} n v, \forall t \in \mathcal{T}(\mathcal{F}): \\
& \forall p \in \mathcal{P}_{m}, p \ll t \wedge\left(\forall p^{\prime} \in \mathcal{P}_{m} \text { s.t. } p^{\prime}<p, p^{\prime} \nless t\right) \Rightarrow \\
& \exists \epsilon^{\prime} \in \mathcal{E} n v,\left\langle\epsilon, \pi_{m}(\ulcorner t\urcorner)\right\rangle \mapsto b s\left\langle\epsilon^{\prime}, \text { accept }_{p}\right\rangle \wedge \Phi\left(\epsilon^{\prime}\right)(p)=t \\
& \wedge\left(\forall p^{\prime} \in \mathcal{P}_{m} \text { s.t. } p^{\prime}<p, \Phi\left(\epsilon^{\prime}\right)\left(p^{\prime}\right) \neq t\right) \\
& \left(\text { Mcomplete }_{O K}\right) \\
& \forall p \in \mathcal{P}_{m}, p \nless t \Rightarrow \exists \epsilon^{\prime} \in \mathcal{E} n v,\left\langle\epsilon, \pi_{m}(\ulcorner t\urcorner)\right\rangle \mapsto_{b s}\left\langle\epsilon^{\prime}, \text { refuse }\right\rangle \\
& \left(\text { Mcomplete }_{K O}\right)
\end{aligned}
$$

A compilation of a pattern $p$ into a program $\pi_{p}$ is said correct, when it is sound and complete.

Property 5 The derivation of a well-formed instruction $i \in\langle$ instr $\rangle$ in an environment $\Gamma, \Delta$, in the extended language, leads either to accept ${ }_{p}$ or refuse, and the reduction is unique.

Proof 6 We proceed by induction over the structure of instructions. The proof is similar to the proof of Property 2, 
We extend the type system presented Figure 2 with the rule:

$$
\frac{\Gamma, \Delta \vdash i_{1}: w f \quad \Gamma, \Delta \vdash i_{2}: w f}{\Gamma, \Delta \vdash i_{1} ; i_{2}: w f}
$$

Let $i=i_{1} ; i_{2}$ be a sequence. By induction, the reduction of $i_{1}$ is unique and leads either to accept ${ }_{p}$ or refuse. In the first case, $\left(s e q_{a}\right)$ is applicable. The reduction of $i_{1} ; i_{2}$ is equal to the reduction of $i_{1}$, so it is unique. In the second case, $\left(\mathrm{seq}_{b}\right)$ is applicable. Since the reduction of $i_{2}$ is unique, the reduction of $i=i_{1} ; i_{2}$ is unique.

Theorem 3 Given a formal anchor \ulcorner\urcorner , $m$ a match construct, and $\pi_{m} \in$ PIL a well-formed program, we have:

$$
\begin{gathered}
\pi_{m} \text { is a correct compilation of } m \\
\Longleftrightarrow \\
\forall \epsilon \in \mathcal{E} n v, \forall t \in \mathcal{T}(\mathcal{F}), \forall p \in \mathcal{P}_{m}: \\
\exists \epsilon^{\prime} \in \mathcal{E} n v,\left\langle\epsilon, \pi_{m}(\ulcorner t\urcorner)\right\rangle \mapsto{ }_{b s}\left\langle\epsilon^{\prime}, \text { accept }_{p}\right\rangle \\
\Leftrightarrow \Phi\left(\epsilon^{\prime}\right)(p)=t \wedge\left(\forall p^{\prime} \in \mathcal{P}_{m} \text { s.t. } p^{\prime}<p, \Phi\left(\epsilon^{\prime}\right)\left(p^{\prime}\right) \neq t\right)
\end{gathered}
$$

Proof 7 We want to show, as in Property 3, that $\left(\right.$ Msound $\left._{O K}\right) \Rightarrow\left(\right.$ Mcomplete $\left._{K O}\right)$ and $\left(\right.$ Mcomplete $\left._{O K}\right) \Rightarrow$ $\left(M\right.$ sound $\left._{K O}\right)$.

In the first case, assume $\left(M\right.$ sound $\left._{O K}\right)$ and $\forall p \in \mathcal{P}_{m}, p \nless t$. Since the reduction of $\left\langle\epsilon, \pi_{m}(\ulcorner t\urcorner)\right\rangle$ is unique, we cannot have a reduction of $\left\langle\epsilon, \pi_{m}(\ulcorner t\urcorner)\right\rangle \mapsto_{b s}\left\langle\epsilon^{\prime}\right.$, accept $\left._{p}\right\rangle$. This reduction exists, hence we have $\left\langle\epsilon, \pi_{m}(\ulcorner t\urcorner)\right\rangle \mapsto_{b s}\left\langle\epsilon^{\prime}\right.$, refuse $\rangle$. The second case can be proved in a similar way.

In order to prove that the compilation $\pi_{m}$ of a match constructs $m$ is correct, we have to consider each statement accept ${ }_{p}$ in the program separately. For each pattern $p$ in the match construct, we build all derivations in $\mapsto_{b s}$ leading to accept ${ }_{p}$, and deduce from it a constraint, formed by a disjunction of conjunctions of single constraints. We can then for each constraint prove the corresponding proof obligation, as expressed in Theorem 3 .

\section{Generating the constraints}

We now describe an algorithm to generate the constraints associated to a PIL program, and discuss the complexity of this algorithm.

\subsection{Algorithm to collect constraints}

The extraction starts with an environment $\epsilon$ instantiating all free variables in the program to verify, as showed in Section 4.2 .

$$
\begin{aligned}
\mathcal{C}(\text { let }(x, u, i), \text { goal })= & \mathcal{C}(i, \text { goal }) \wedge x=u \\
\mathcal{C}\left(\text { if }\left(e, i_{1}, i_{2}\right), \text { goal }\right)= & \left(\mathcal{C}\left(i_{1}, \text { goal }\right) \wedge e \equiv \text { true }\right) \\
& \left.\vee \mathcal{C}\left(i_{2}, \text { goal }\right) \wedge e \equiv \text { false }\right) \\
\mathcal{C}\left(i_{1} ; i_{2}, \text { goal }\right)= & \mathcal{C}\left(i_{1}, \text { goal }\right) \vee\left(\mathcal{C}\left(i_{1}, \text { refuse }\right) \wedge \mathcal{C}\left(i_{2}, \text { goal }\right)\right) \\
\mathcal{C}(i, \text { goal }) & \quad \top \text { if } i=\text { goal }, \\
& \perp \text { otherwise }
\end{aligned}
$$

The algorithm computes a disjunction of conjunction of constraints. The disjuction represents the different path the control flow can take in the program, while the conjunctions represents the set of constraints raised in one of those path.

It is interesting to note that in the case of simple patterns, when we do not consider the ; instruction, and when there is only one occurence of accept in the program, then only one of such path is possible for 
the control flow to reach accept, and so all disjuctions can be simplified by a simple boolean analysis of the generated constraint.

This function is too abstract to be used in this form, since we need for building the proof obligations of the correctness theorem the substitution built by the program when reaching accept. To ease the implementation we allow us to pass to the constraint extraction function the substitution built by the evaluation, and apply it to the constraints where possible.

$$
\begin{aligned}
\mathcal{C}(\epsilon, \text { let }(x, u, i), \text { goal })= & \mathcal{C}(\epsilon[x \leftarrow u], i, \text { goal }) \\
\mathcal{C}\left(\epsilon, \text { if }\left(e, i_{1}, i_{2}\right), \text { goal }\right)= & \left(\mathcal{C}\left(\epsilon, i_{1}, \text { goal }\right) \wedge \epsilon(e) \equiv \text { true }\right) \\
& \vee\left(\mathcal{C}\left(\epsilon, i_{2}, \text { goal }\right) \wedge \epsilon(e) \equiv \text { false }\right) \\
\mathcal{C}\left(\epsilon, i_{1} ; i_{2}, \text { goal }\right) & =\mathcal{C}\left(\epsilon, i_{1}, \text { goal }\right) \vee\left(\mathcal{C}\left(\epsilon, i_{1}, \text { refuse }\right) \wedge \mathcal{C}\left(\epsilon, i_{2}, \text { goal }\right)\right) \\
\mathcal{C}(\epsilon, i, \text { goal }) & =\top \text { if } i=\text { goal }, \perp \text { otherwise }
\end{aligned}
$$

In the resulting set of constraints $C$, we propagate variable instantiations, and apply the formal anchor equations to simplify the constraints.

In practice, this simplification is done during the extraction of the constraints, to allow detecting unsatisfiable sets of constraints (denoting an impossible path in the program flow) as early as possible, and discarding them.

\subsection{Simple example}

As an example, let us consider the pattern $g(x, b)$, with $x \in \mathcal{X}$. Let us now suppose that our compiler produces the following program, where $\mathrm{s}$ is an input variable:

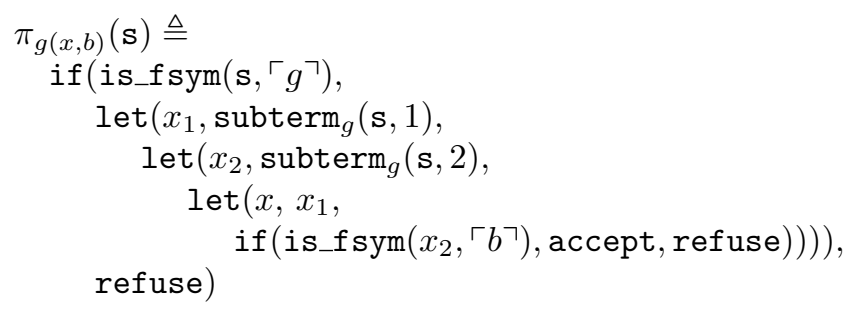

We have to start the constraint extraction with $\epsilon=[\mathrm{s} \leftarrow t]$, and want to derive accept.

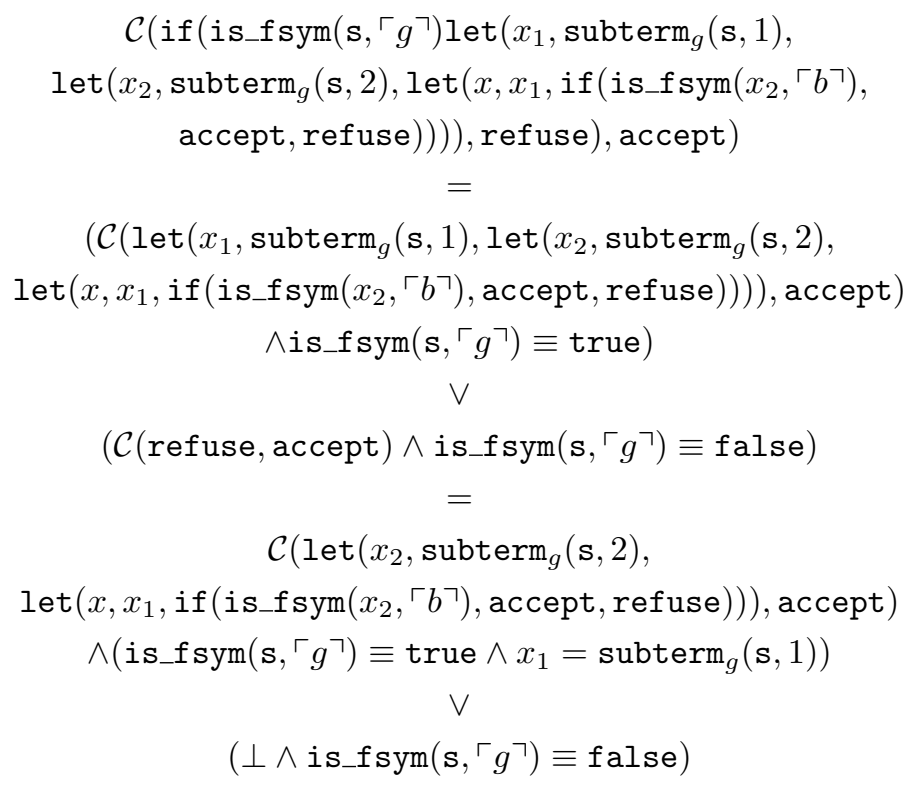




$$
\begin{aligned}
& = \\
& \mathcal{C}\left(\operatorname{let}\left(x, x_{1}, \operatorname{if}\left(\text { is_f } \operatorname{sym}\left(x_{2},\ulcorner b\urcorner\right) \text {, accept, refuse }\right)\right) \text {, accept }\right) \\
& \left.\wedge \text { is_fsym }(\mathbf{s},\ulcorner g\urcorner) \equiv \operatorname{true} \wedge x_{1}=\operatorname{subterm}_{g}(\mathbf{s}, 1) \wedge x_{2}=\operatorname{subterm}_{g}(\mathrm{~s}, 2)\right) \\
& \checkmark \\
& (\perp \wedge \text { is_f } \operatorname{sym}(\mathbf{s},\ulcorner g\urcorner) \equiv \text { false }) \\
& = \\
& \mathcal{C}\left(\text { if (is_f } \operatorname{sym}\left(x_{2},\ulcorner b\urcorner\right)\right. \text {, accept, refuse), accept) } \\
& \wedge \text { is_fsym }(\mathbf{s},\ulcorner g\urcorner) \equiv \text { true } \wedge x_{1}=\operatorname{subterm}_{g}(\mathbf{s}, 1) \\
& \left.\wedge x_{2}=\text { subterm }_{g}(\mathrm{~s}, 2) \wedge x=x_{1}\right) \\
& \checkmark \\
& (\perp \wedge \text { is_fsym }(\mathbf{s},\ulcorner g\urcorner) \equiv \text { false }) \\
& = \\
& \text { ( } \mathcal{C} \text { (accept, accept) } \\
& \wedge \text { is_f } \operatorname{sym}(\mathbf{s},\ulcorner g\urcorner) \equiv \text { true } \wedge x_{1}=\operatorname{subterm}_{g}(\mathrm{~s}, 1) \\
& \left.\wedge x_{2}=\operatorname{subterm}_{g}(\mathrm{~s}, 2) \wedge x=x_{1} \wedge \text { is_f } \operatorname{sym}\left(x_{2},\ulcorner b\urcorner\right) \equiv \text { true }\right) \\
& \mathrm{V} \\
& (\mathcal{C} \text { (refuse, accept) } \\
& \wedge \text { is_fsym }(\mathbf{s},\ulcorner g\urcorner) \equiv \text { true } \wedge x_{1}=\operatorname{subterm}_{g}(\mathbf{s}, 1) \\
& \left.\wedge x_{2}=\operatorname{subterm}_{g}(\mathrm{~s}, 2) \wedge x=x_{1} \wedge \text { is_f } \operatorname{sym}\left(x_{2},\ulcorner b\urcorner\right) \equiv \text { false }\right) \\
& \checkmark \\
& (\perp \wedge \text { is_fsym }(\mathbf{s},\ulcorner g\urcorner) \equiv \text { false }) \\
& = \\
& \left(\top \wedge \text { is_fsym }(\mathbf{s},\ulcorner g\urcorner) \equiv \operatorname{true} \wedge x_{1}=\operatorname{subterm}_{g}(\mathbf{s}, 1) \wedge x_{2}=\operatorname{subterm}_{g}(\mathbf{s}, 2)\right. \\
& \left.\wedge x=x_{1} \wedge \text { is_f } \operatorname{sym}\left(x_{2},\ulcorner b\urcorner\right) \equiv \text { true }\right) \\
& \checkmark \\
& \left(\perp \wedge \text { is_fsym }(\mathbf{s},\ulcorner g\urcorner) \equiv \operatorname{true} \wedge x_{1}=\operatorname{subterm}_{g}(\mathrm{~s}, 1)\right. \\
& \left.\wedge x_{2}=\operatorname{subterm}_{g}(\mathrm{~s}, 2) \wedge x=x_{1} \wedge \text { is_f } \operatorname{sym}\left(x_{2},\ulcorner b\urcorner\right) \equiv \text { false }\right) \\
& \mathrm{V} \\
& (\perp \wedge \text { is_f } \operatorname{sym}(\mathbf{s},\ulcorner g\urcorner) \equiv \text { false })
\end{aligned}
$$

\subsection{Simplifying constraints}

Using the algorithm to collect constraints produces a huge formula, containing many $\top$ and $\perp$, so we can first simplify this formula as a boolean formula.

We apply the following rewrite system, with a letftmost-innermost strategy:

$$
\begin{array}{ll}
\perp \wedge x & \rightarrow \perp \\
x \wedge \perp & \rightarrow \\
\top \vee x & \rightarrow \top \\
x \vee \top & \rightarrow \top \\
\perp \vee x & \rightarrow x \\
x \vee \perp & \rightarrow x \\
\neg \top & \rightarrow \perp \\
\neg \perp & \rightarrow
\end{array}
$$


This rewrite system simplify the boolean constraints, to obtain the simplified contraints.

The extracted constraints for $\pi_{g(x, b)}$ are:

$$
\begin{gathered}
\text { is_fsym }(\mathrm{s},\ulcorner g\urcorner) \equiv \text { true }, x_{1}=\text { subterm }_{g}(\mathrm{~s}, 1) \\
\wedge \quad x_{2}=\text { subterm }_{g}(\mathrm{~s}, 2) \\
\wedge \quad x=x_{1} \\
\wedge \quad \text { is_f } \operatorname{sym}\left(x_{2},\ulcorner b\urcorner\right) \equiv \text { true }
\end{gathered}
$$

Those constraints can be simplified using the definitions of the mapping,

$$
\begin{aligned}
\operatorname{eq}\left(\left\ulcorner t_{1}\right\urcorner,\left\ulcorner t_{2}\right\urcorner\right) & \equiv\left\ulcorner t_{1}=t_{2}\right\urcorner \\
\text { is_fsym }(\ulcorner t\urcorner,\ulcorner f\urcorner) & \equiv\ulcorner\mathcal{S} y m b(t)=f\urcorner \\
\text { subterm }_{f}(\ulcorner t\urcorner,\ulcorner i\urcorner) & \equiv\left\ulcorner t_{\mid i}\right\urcorner \text { if } \mathcal{S} y m b(t)=f
\end{aligned}
$$

The mapping equalities can be oriented, to be used as a rewrite system. Informally, the goal is to transform a constraint giving information about the objects manipulated by the program into a constraint giving information about the algebraic terms used at source level.

$$
\begin{aligned}
\operatorname{eq}\left(\left\ulcorner t_{1}\right\urcorner,\left\ulcorner t_{2}\right\urcorner\right) & \rightarrow\left\ulcorner t_{1}=t_{2}\right\urcorner \\
\text { is_fsym }(\ulcorner t\urcorner,\ulcorner f\urcorner) & \rightarrow\ulcorner\mathcal{S} y m b(t)=f\urcorner \\
\text { subterm }_{f}(\ulcorner t\urcorner,\ulcorner i\urcorner) & \rightarrow\left\ulcorner t_{\mid i}\right\urcorner \text { if } \mathcal{S} y m b(t)=f
\end{aligned}
$$

Applying this rewrite system to the constraints generated for $\pi_{g(x, b)}$ produces the constraint:

$$
\begin{gathered}
\operatorname{Symb}(\mathbf{s})=g \\
\wedge x_{1}=\mathbf{s}_{\mid 1} \\
\wedge x_{2}=\mathbf{s}_{\mid 2} \\
\wedge x=x_{1} \\
\wedge \\
\mathcal{S} y m b\left(x_{2}\right)=b
\end{gathered}
$$

\subsection{Size of the generated formula}

Let us consider the size of the input as the number of nodes of the input abstract syntax tree.

All rules except the extraction rule for the sequence ; are linear, each node in the input abstract syntax tree is visited once.

The rule for sequence visits the left subtree of ; twice, thus leading to an exponential in the number of ; nodes in the input. However, the sequence ; is associative, so we can consider that the left subtree of a sequance is not itself a sequence, and the generated code usually do not contain many nested levels of sequences, so this exponential do not appear in practical use.

\subsection{Decidability}

The theorems we want to prove have their only quantifiers at the root, introducing the input variables.

They correspond to the second part or the correctness theorem [] and if they hold, prove that the compilation of the corresponding patterns was successful. $\mathcal{C} \pi_{p}$ is the constraint formula produced by the application of the constraint extraction algorithm on the PIL program $\pi_{p}$, corresponding to the compilation of the matching problem $p . \mathcal{C} \pi_{p}(t)$ represents the fact that the term $t$ satisfy this constraint formula.

Theorem 4 Given a formal anchor $\left\ulcorner\right.$, a pattern $p \in \mathcal{T}(\mathcal{F}, \mathcal{X})$, and a well-formed program $\pi_{p} \in \mathrm{PIL}$, we have:

$$
\begin{aligned}
& \pi_{p} \text { is a correct compilation of } p \\
& \forall t \in \mathcal{T}(\mathcal{F}), \mathcal{C} \pi_{p}(t) \Leftrightarrow \exists \epsilon^{\prime} \in \mathcal{E} n v, \Phi\left(\epsilon^{\prime}\right)(p)=t
\end{aligned}
$$


The part we want to prove automatically is:

$$
\forall t \in \mathcal{T}(\mathcal{F}), \mathcal{C} \pi_{p}(t) \Leftrightarrow \exists \epsilon^{\prime} \in \mathcal{E} n v, \Phi\left(\epsilon^{\prime}\right)(p)=t
$$

Since the output substitution can be computed from the extracted constraints (and directly, using the second extraction algorithm), we can also remove from the property we have to prove the existential quantifier, and use this computed substitution in place of $\epsilon^{\prime}$.

The property to prove becomes:

$$
\forall t \in \mathcal{T}(\mathcal{F}), \mathcal{C} \pi_{p}(t) \Leftrightarrow \Phi(\epsilon)(p)=t
$$

Proving that: $\forall t \in \mathcal{T}(\mathcal{F}), \Phi(\epsilon)(p)=t \Rightarrow \mathcal{C} \pi_{p}(t)$ can be done by orienting the axioms defining the subterm and symbol property.

\section{$7 \quad$ Early Experimental Results}

The presented work has been implemented and applied to the intermediate language of Tom [?]. Tom is a language extension which adds pattern matching primitives to C, Java, and Caml. One particularity is to provide support for matching modulo sophisticated theories, like associative operators with neutral element. However, in this work, we only considered the case of the empty theory (i.e. syntactic matching), with possibly non-linear patterns.

Tom is based on the notion of formal anchor presented in Section 2.3. Thus, it is data structure independent, and customizable for any term implementation. Considering a simple term implementation in C, for example, we can define the following anchor:

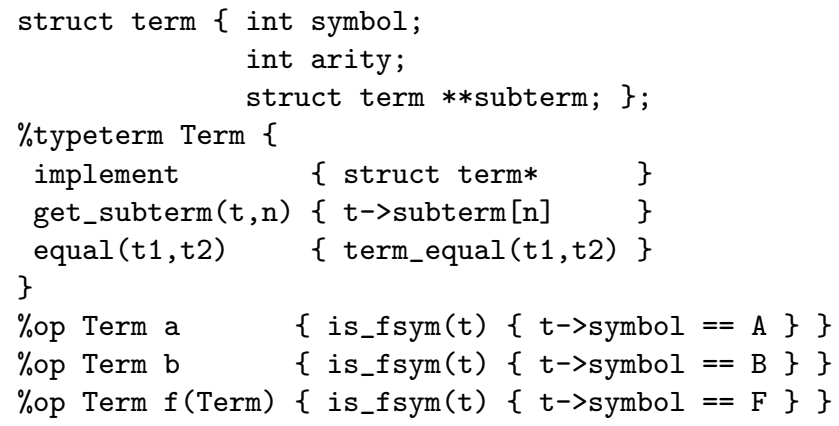

Given a \%match construct, as illustrated by Figure 6, the compiler translates patterns into PIL instructions, which use the previously defined formal anchor. In practice, this mapping is supposed correct, in the sense that structural properties of terms should be preserved. To simplify this task, when no particular data-structure is required, a generator of term based implementations, coupled with a generator of formal anchors, can be used [?].

To prove the generated PIL code correct, we recently added to Tom a component (constraints extractor) which generates, for each pattern $p$, the constraints $\mathcal{C} \pi_{p}$ and $\mathcal{C} p, \sigma=(\exists \sigma, \sigma(p)=t)$, where $t$ is the input term. In a second step, these two constraints are sent to a prover to show their equivalence. To experiment our approach, we used Zenon, because, in addition to be fully automatic, it can generate a Coq formal proof when it succeeds. This is essential in our "skeptical" approach since it allows the user of the generated program to verify the proof by himself.

The verification tool is integrated into the Tom architecture, but note that no support from the internal compiler is needed: $\mathcal{C} p, \sigma$ are extracted from the AST produced by the parser, and $\mathcal{C} \pi_{p}$ are extracted from the PIL program produced by the compiler, or any other component such as an optimizer for example. Seeing the compiler as a black-box allows us to perform any kind of optimization unless PIL code is generated. At the moment we handle only the intermediate code of the compiler, ignoring the parser and the code generator. 


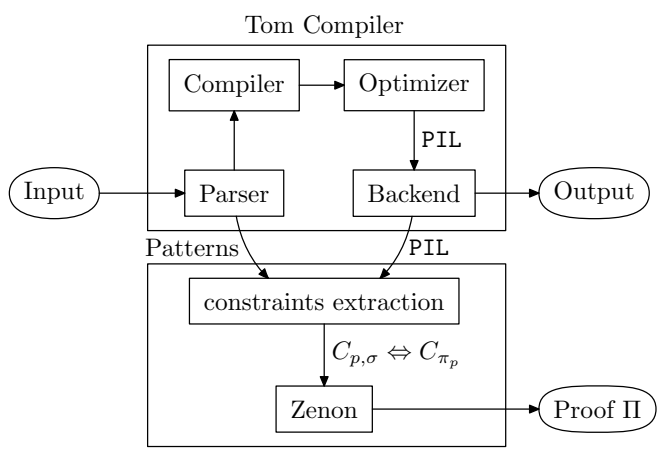

Figure 6: Global architecture of Tom

In our case, the backend performs a so straightforward one-to-one translation, from PIL instructions to host language instructions, that we trust in its correctness.

The interest of this approach is to allow to verify code corresponding to an optimized many-to-one algorithm, where common tests are factored.

To illustrate the applicability of the present approach, we tested our validator on several small examples, all of which worked with success, in an efficient way. For a more realistic test, to show how the approach scales to biggest problems, we generated proof obligations corresponding to the compilation of Tom itself (written in Tom). 184 patterns were extracted by the parser, after discarding associative patterns. From this set of patterns, 1018 applications of inference rules were needed to compute all derivations which lead to accept. This step generated 834 constraints $\left(\mathcal{C} \pi_{p}\right)$. Most of them were tautologies of the form $\epsilon\left(\operatorname{subterm}_{g}(\mathbf{s}, 1)\right) \equiv \mathbf{s}_{\mid 1}$. After a first step of simplification, 273 remaining constraints were simplified using 2533 三-equivalence relation steps. On a PowerMac G5 (2GHz), the compilation of Tom, with the generation of theorems to prove, only increased the compilation time by $20 \%$ (going from 70 seconds to 84 seconds). This clearly shows that the approach can scale to large applications. In the current implementation, the translation to Zenon formalism is done fully automatically starting from the Tom program.

\section{Conclusion and future works}

When using a compiler, we always think it is correct. When writing a compiler, we know it is incorrect. This drives us to present a framework addressing the specific problem of generated pattern matching code validation. The main benefit of such an approach over a traditional compiler is that the compiler output is formally checked after each compilation, thus simplifying testing and development and providing a way to prove the formal validity of the generated code. We have seen that the proposed approach is powerful and flexible enough to validate the compilation of match constructs à la Caml or Tom.

We are now attacking the challenging problem of extending this method to support matching with associative (list) operators, like those of the Tom language, and with associative-commutative operators, like in many rewriting based languages like ELAN. Matching modulo theories is much more elaborated than syntactic matching, and so is writing such a pattern matching compiler. Validation of the produced code can then help developing and debugging new optimizations for these matching theories. Furthermore, when matching modulo theories, a new completeness problem has to be solved: the generated matching code has not only to find a substitution if the matching problem has one, but may have to produce all possible solutions for the matching problem.

The approach proposed here generates proof obligations of a very strict form. These proof obligations are in general easy to prove, but we should investigate our current conjecture that this class of problems is indeed decidable.

Although we were only interested in this work in the correctness of the generated code against the source 
problem, some additional properties of the source system could be proved by this method. For example the completeness of definition of a function defined by pattern matching could be proved by showing that there is no possible reduction to refuse.

Finally, our ultimate goal is to formally prove the correct compilation of the normalization process induced by a rewrite system. Proving the correct compilation of rewrite system execution will allow us to safely deduce on the program produced by the compilation of a rewriting specification the properties proved for this specification, like termination or confluence. This paper is a first but crucial step in this direction.

Acknowledgments: We would like to thank Luigi Liquori for his inside full reading on a preliminary version of this paper, Pierre Weis and Germain Faure for useful comments on this work. Special thanks are due to Damien Doligez for fruitful discussions and his help in connecting our tool to Zenon. We also thank the anonymous referees for valuable comments and suggestions that led to a substantial improvement of the paper.

\section{References}

[1] Franz Baader and Tobias Nipkow. Term Rewriting and all That. Cambridge University Press, 1998.

[2] Don Batory, Bernie Lofaso, and Yannis Smaragdakis. JTS: tools for implementing domain-specific languages. In Proceedings Fifth International Conference on Software Reuse, pages 143-153, Victoria, BC, Canada, 1998. IEEE.

[3] Robert S. Boyer and Yuan Yu. Automated correctness proofs of machine code programs for a commercial microprocessor. In D. Kapur, editor, Proceedings of the Eleventh International Conference on Automated Deduction, pages 416-430. Springer-Verlag, 1992.

[4] Horatiu Cirstea, Claude Kirchner, and Luigi Liquori. Matching Power. In Aart Middeldorp, editor, Proceedings of RTA'2001, volume 2051 of LNCS, Utrecht (The Netherlands), May 2001. Springer-Verlag.

[5] Damien Doligez. Zenon: an automatic theorem prover for first-order logic. Available as part of the Focal system at http://focal.inria.fr/download.

[6] Gilles Dowek, Thérèse Hardin, and Claude Kirchner. Theorem proving modulo. Journal of Automated Reasoning, 31(1):33-72, Nov 2003.

[7] Fabrice Le Fessant and Luc Maranget. Optimizing pattern matching. In Proceedings of the sixth ACM SIGPLAN International Conference on Functional Programming, pages 26-37. ACM Press, 2001.

[8] Sumit Gulwani and George C. Necula. Global value numbering using random interpretation. In $P O P L$ '04: Proceedings of the 31st ACM SIGPLAN-SIGACT symposium on Principles of programming languages, pages 342-352. ACM Press, 2004.

[9] Gilles Kahn. Natural semantics. In 4th Annual Symposium on Theoretical Aspects of Computer Sciences on STACS 87, pages 22-39, London, UK, 1987. Springer-Verlag.

[10] Hélène Kirchner and Pierre-Etienne Moreau. Promoting rewriting to a programming language: A compiler for non-deterministic rewrite programs in associative-commutative theories. Journal of Functional Programming, 11(2):207-251, 2001.

[11] Andreas Krall and Jan Vitek. On extending java. In Proceedings of the Joint Modular Languages Conference on Modular Programming Languages, pages 321-335. Springer-Verlag, 1997.

[12] David Lacey, Neil D. Jones, Eric Van Wyk, and Carl Christian Frederiksen. Proving correctness of compiler optimizations by temporal logic. In Proc. 29th ACM Symposium on Principles of Programming Languages, pages 283-294. ACM Press, 2002. 
[13] John McCarthy and James Painter. Correctness of a compiler for arithmetic expressions. In J. T. Schwartz, editor, Proceedings Symposium in Applied Mathematics, Vol. 19, pages 33-41. AMS, 1967.

[14] Pierre-Etienne Moreau, Christophe Ringeissen, and Marian Vittek. A Pattern Matching Compiler for Multiple Target Languages. In G. Hedin, editor, 12th Conference on Compiler Construction, volume 2622 of $L N C S$, pages 61-76. Springer-Verlag, May 2003.

[15] F. Lockwood Morris. Advice on structuring compilers and proving them correct. In Proceedings of the 1st annual ACM SIGACT-SIGPLAN Symposium on Principles of Programming Languages, pages 144-152. ACM Press, 1973.

[16] George C. Necula. Proof-carrying code. In Proceedings of the 24th ACM SIGPLAN-SIGACT Symposium on Principles of Programming Languages, pages 106-119. ACM Press, 1997.

[17] George C. Necula and Peter Lee. The design and implementation of a certifying compiler. SIGPLAN Not., 39(4):612-625, 2004.

[18] Dino P. Oliva, John D. Ramsdell, and Mitchell Wand. The VLISP verified PreScheme compiler. Lisp Symb. Comput., 8(1-2):111-182, 1995.

[19] Amir Pnueli, Michael Siegel, and Eli Singerman. Translation validation. In Proceedings of the 4th International Conference on Tools and Algorithms for Construction and Analysis of Systems, pages 151-166. Springer-Verlag, 1998.

[20] Martin C. Rinard and Darko Marinov. Credible compilation with pointers. In Proceedings of the FLoC Workshop on Run-Time Result Verification, Trento, Italy, July 1999.

[21] Xavier Rival. Symbolic transfer function-based approaches to certified compilation. In Proceedings of the 31st ACM SIGPLAN-SIGACT symposium on Principles of programming languages, pages 1-13. ACM Press, 2004.

[22] Mark. G. J. van den Brand, Pierre-Etienne Moreau, and Jurgen Vinju. Generator of efficient strongly typed abstract syntax trees in Java. IEE Proceedings - Software Engineering, 152(2):70-79, April 2005.

[23] Philip Wadler. Views: a way for pattern matching to cohabit with data abstraction. In Proceedings of the 14th ACM SIGACT-SIGPLAN Symposium on Principles of Programming Languages, pages 307-313. ACM Press, 1987.

[24] Dinghao Wu, Andrew W. Appel, and Aaron Stump. Foundational proof checkers with small witnesses. In Proceedings of the 5th ACM SIGPLAN international Conference on Principles and Practice of Declarative Programming, pages 264-274. ACM Press, 2003. 